\title{
Validation of a new sensor to measure the concentration of polyethylene glycol for unsaturated soil testing
}

\author{
Buzzi $^{1, a}$ O., Routley ${ }^{2}$ B., Yuan ${ }^{1}$ S. \\ ${ }^{1}$ Priority Research Centre for Geotechnical and Materials Modelling, School of Engineering, The University of Newcastle, NSW, Australia. \\ ${ }^{2}$ School of Electrical Engineering and Computer Science, University of Newcastle, Callaghan, Australia
}

\begin{abstract}
Accurate measurement of PEG concentration is primordial when using the osmotic method to control soil suction. Because PEG is an inert molecule, common chemistry methods are not adequate to measure its concentration and researchers resort to the measurement of Brix Index. Such measurement can be manual, which is not very accurate and time consuming but cheap. On the other hand, more advanced sensors do exist but they tend to be quite expensive. This paper presents a new sensor for the indirect measurement of PEG concentration based on a tuneable diode laser and fibre optics. The laser signal is shone through the PEG and the reduction in signal voltage is correlated to the PEG concentration. The system has been assessed for temperature stability and accuracy. Such sensor offers the possibility of multipoint measurement, which significantly reduces the capital cost of the equipment.
\end{abstract}

\section{Introduction}

Accurate and reliable control of suction is of paramount importance to produce high quality data in experimental unsaturated soil mechanics. The osmotic technique is one of the methods available to control soil suction [1-3]. The technique relies on a semi permeable membrane separating a solution of dissolved polyethylene glycol (PEG) and the soil specimen to be tested. The difference of PEG concentration across the membrane generates suction within the soil specimen. The osmotic technique is relatively simple to use and works for suctions up to 9 or $10 \mathrm{MPa}$, which is well beyond the capability of the axis translation technique (i.e. 1.5 MPa), another method allowing suction control. One technical challenge with the osmotic method resides in the measurement of the PEG concentration. Indeed, PEG is an inert molecule and, consequently, common chemistry methods cannot be used. A simple alternative is to measure the refractive index, also called Brix index, of the PEG solution. Indeed, the Brix index is directly correlated to the concentration and is independent of the PEG molecular weight [4]. Brix Index measurement can be done with hand held refractometers, which is not very costly but not very accurate, or using an automatic in line process refractometer, which is quite an expensive piece of equipment. This study aims at developing and validating a new sensor for PEG concentration. The sensor is based on the measurement of absorbance of the PEG solution using a tunable diode laser and a photodetector. The present paper presents the proof of concept and an estimation of errors and temperature sensitivity.

\section{Experimental setup}

The experimental setup is presented in Figure 1. Note that, in this validation phase, the measurements were not made in situ. Instead, some PEG solutions (details given in Table 1) were placed in micro cuvettes and a near infrared laser was shone through the cuvette and the PEG solution. A controller (Model ITC4001 from Thorlabs) was used to supply the laser with a constant power of 42 $\mathrm{mA}$. The laser beam was transported via a fibre optic to the cuvette holder where the cuvettes containing the PEG were placed. A photodetector (Thorlabs model PDA 50BEC) was then used to record the voltage of the output signal, required to compute the absorbance $A$ :

$$
A=\log _{10}\left(I_{0} / I\right)
$$

where $I_{o}$ and $I$ are the input and output voltage, respectively. Note that this absorbance actually characterises the system (cuvette + PEG) since the laser beam shines through $8.5 \mathrm{~mm}$ of glass and 4 of PEG solution. Note also that $A$ has no unit. The whole setup, but the computer, was placed in a laboratory fridge for a control of temperature at \pm 0.1 degree Celsius.

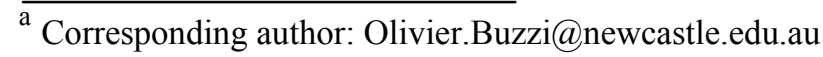




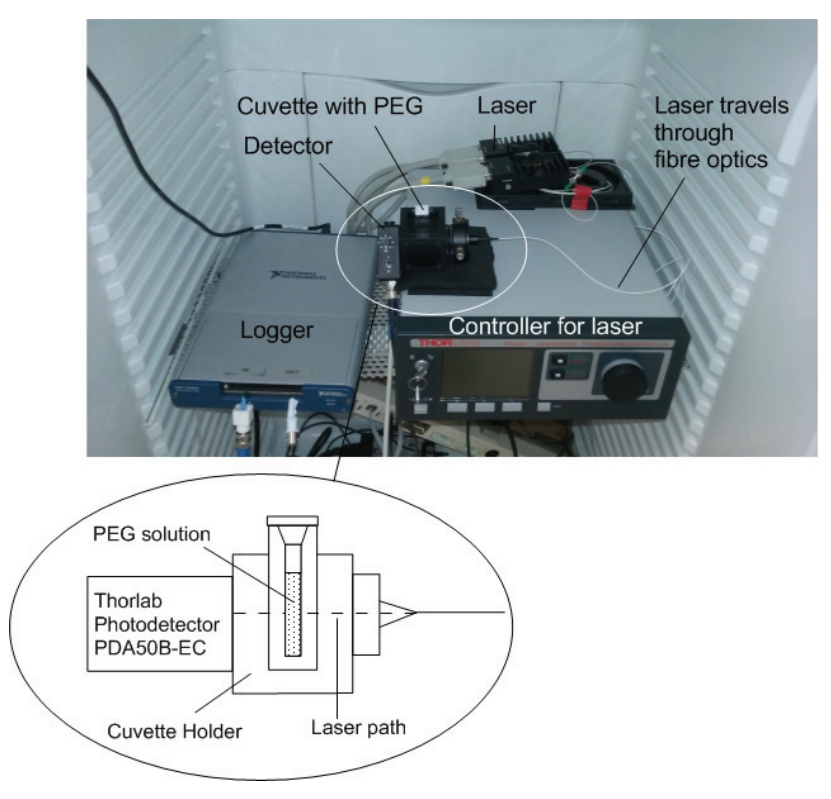

Figure 1. Sketch of the experimental setup used to validate the new sensor.

\section{Experimental program}

The absorbance of a number of PEG solutions was measured using the setup described in Figure 1. First, the existence of a correlation between absorbance and PEG concentration was investigated using three PEG types (PEG6000, 20000 and 35000 noted PEG6k, 20k and 35k, respectively) and up to 6 concentrations (test series \#1). The range of concentration corresponds to suction values ranging from about $30 \mathrm{kPa}$ to about $3 \mathrm{MPa}$ (using $s=11$ $\left.c^{2},[2]\right)$. These tests were conducted at 20 degrees. Then, a series of tests were conducted to assess the repeatability of measurement, accuracy and possible drift (test series $\# 2$ ). A third series (\#3) focused on the sensitivity to temperature. For these three series of tests, the solutions were prepared by dissolving a determined mass of PEG in about $20 \mathrm{~mL}$ of deionised water. Masses were recorded using a high precision scale (to the $1 / 10,000^{\text {th }}$ of a gram), hence minimizing the error on the concentration.

Table 1. Experimental program. All concentrations $C$ are in grams of PEG per grams of water.

\begin{tabular}{|c|c|c|c|c|}
\hline & $\mathrm{T}\left[{ }^{\circ} \mathrm{C}\right]$ & PEG6k & PEG20k & PEG35k \\
\hline $\begin{array}{l}\text { Series 1: } \\
\text { Correlation } \\
\text { Concentration } \\
\text { - Absorbance }\end{array}$ & 20 & $\begin{array}{l}\mathrm{C}= \\
0.05, \\
0.1,0.2, \\
0.3,0.4, \\
0.5\end{array}$ & $\begin{array}{l}\mathrm{C}= \\
0.05 \\
0.1,0.2 \\
0.3,0.4 \\
0.5\end{array}$ & $\begin{array}{l}\mathrm{C}= \\
0.05, \\
0.1,0.2, \\
0.3,0.4\end{array}$ \\
\hline $\begin{array}{l}\text { Series 2: } \\
\text { Repeatability, } \\
\text { drift, detection }\end{array}$ & 20 & & $\begin{array}{l}\mathrm{C}= \\
0.05,0.5\end{array}$ & \\
\hline $\begin{array}{l}\text { Series 3: } \\
\text { Effect of } \\
\text { temperature }\end{array}$ & $\begin{array}{l}20, \\
23, \\
26,29\end{array}$ & & $\begin{array}{l}\mathrm{C}= \\
0.05,0.5\end{array}$ & \\
\hline $\begin{array}{l}\text { Series 4: } \\
\text { Effect of } \\
\text { chemical } \\
\text { species }\end{array}$ & 20 & & $\begin{array}{l}\mathrm{C}= \\
0.05,0.5\end{array}$ & \\
\hline
\end{tabular}

A fourth series investigates the possibility of interference in the measurement should there be other chemical species in solution. Tadza [5] showed that some of the cations and anions naturally present in soils can diffuse in the PEG solution. Considering the wide range of chemical species and concentrations in natural soils, it has been decided to dissolve PEG in synthetic seawater (composition given in Table 2) and check whether the absorbance measurement is affected by the presence of the chemical species.

Table 2. Chemical composition of artificial seawater used for this study. Concentrations in $\mathrm{g} / \mathrm{L}$

\begin{tabular}{|c|c||c|c|}
\hline $\mathrm{NaCl}$ & 24.53 & $\mathrm{NaHCO}_{3}$ & 0.201 \\
\hline $\mathrm{MgCl}_{2}$ & 5.2 & $\mathrm{KBr}$ & 0.101 \\
\hline $\mathrm{Na}_{2} \mathrm{SO}_{4}$ & 4.09 & $\mathrm{H}_{3} \mathrm{BO}_{3}$ & 0.027 \\
\hline $\mathrm{CaCl}_{2}$ & 1.16 & $\mathrm{~S}_{\mathrm{r}} \mathrm{Cl}_{2}$ & 0.025 \\
\hline $\mathrm{KCl}$ & 0.695 & $\mathrm{NaF}$ & 0.003 \\
\hline
\end{tabular}

\section{Results}

\subsection{Test series \#1}

Figure $2 \mathrm{a}$ depicts the evolution of absorbance in time for one concentration of PEG 20k. It shows that the equilibrium is not instantaneous; it takes some minutes to reach equilibrium. Similar curves were observed for all tests and data were recorded after equilibrium was reached. Figure $2 \mathrm{~b}$ shows the absorbance measurements pertaining to the three PEG types and whole range of concentration. It can clearly be seen that the higher the PEG concentration, the lower the absorbance and the correlation can reasonably be considered linear. It is also remarkable that, for a given concentration, the absorbance value is independent from the PEG molecular mass.

Optical absorption is theoretically described using Beer Lambert's law which states that the absorbance is proportional to the concentration of attenuating species:

$$
A=a \times b \times c
$$

where $A$ is the absorbance, $a$ is the attenuation coefficient, $b$ is the path length and $c$ is the concentration of attenuating species.

Here, the laser wavelength was chosen so that the laser beam be absorbed by the water rather than the PEG. In other words, water is the attenuating species and the experimental results are fully consistent with Beer Lambert's law. Indeed, as the PEG concentration increases, the water concentration (in grams of water per grams of solution) decreases and the absorbance decreases. 
The results presented in Figure $2 b$ validate the idea of inferring PEG concentration from absorbance measurement.

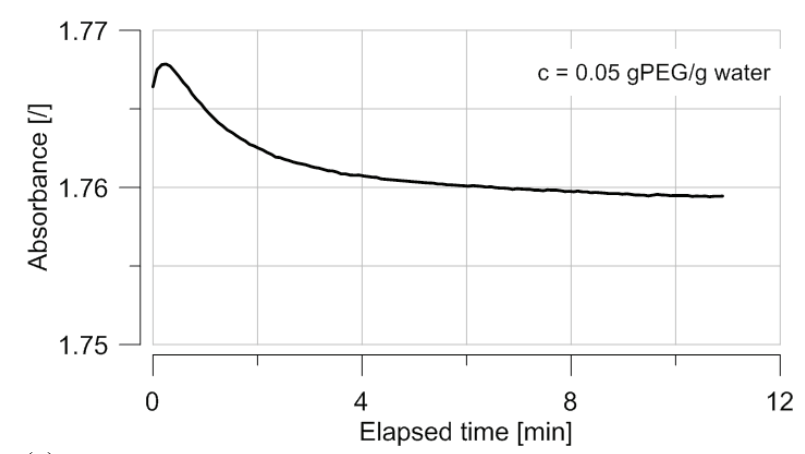

(a)

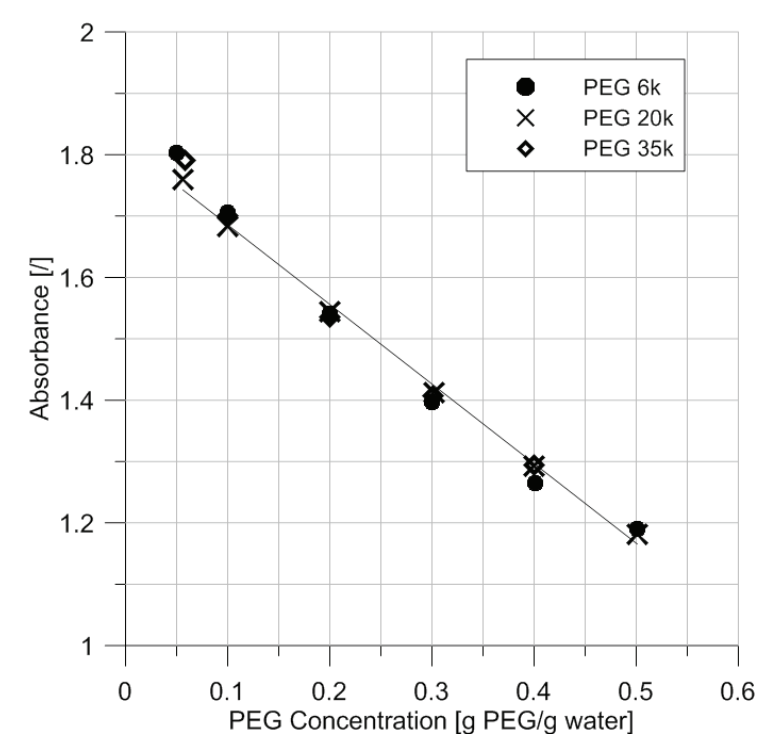

(b)

Figure 2. (a): evolution of absorbance with time for a PEG20k at $0.05 \mathrm{~g} \mathrm{PEG} / \mathrm{g}$ water. (b): Results of absorbance values (at equilibrium) for different concentrations of PEG6K, 20k and PEG35k.

\subsection{Test series \#2}

This section deals with the estimation of errors on the absorbance measurement and on the estimation of PEG concentration. Several sources of uncertainties have been estimated, as summarised below:

-At any point in time, the resolution is 0.001 [no unit], yielding an absolute error of $\Delta A_{\text {resolution }}= \pm 0.0005$

-A repeatability exercise showed that the largest scattering ( $\max$ value $-\min$ value $=0.009$ [no unit]) occurs at the highest concentration ( $0.5 \mathrm{~g}$ PEG/ $\left.\mathrm{g} \mathrm{H}_{2} \mathrm{O}\right)$, yielding an absolute error of $\Delta A_{\text {repeat }}=\mp 0.0045$ [no unit].

-A drift exercise (continuous measurement of the same solution for 2 days) showed an amplitude of fluctuation ( $\max$ value - min value) of about 0.007 [no unit], yielding an absolute error of $\Delta A_{\text {drift }}=\mp 0.0035$
These uncertainties are independent and an equivalent uncertainty, referred to as the equivalent resolution of the sensor, can be estimated as:

$$
\begin{aligned}
\Delta A & =\sqrt{\Delta A_{\text {resolution }}^{2}+\Delta A_{\text {repeat }}^{2}+\Delta A_{\text {drift }}^{2}} \\
\Delta A & = \pm 0.0057
\end{aligned}
$$

Note that the first drift exercise was conducted with the PEG solution in the laboratory fridge but the laser was kept outside the fridge. Significant variations were observed, which can be attributed to room temperature fluctuation (See Figure 3). Following this, the whole setup was placed in the temperature-controlled environment. This proved to reduce the fluctuation to a magnitude of 0.007 .
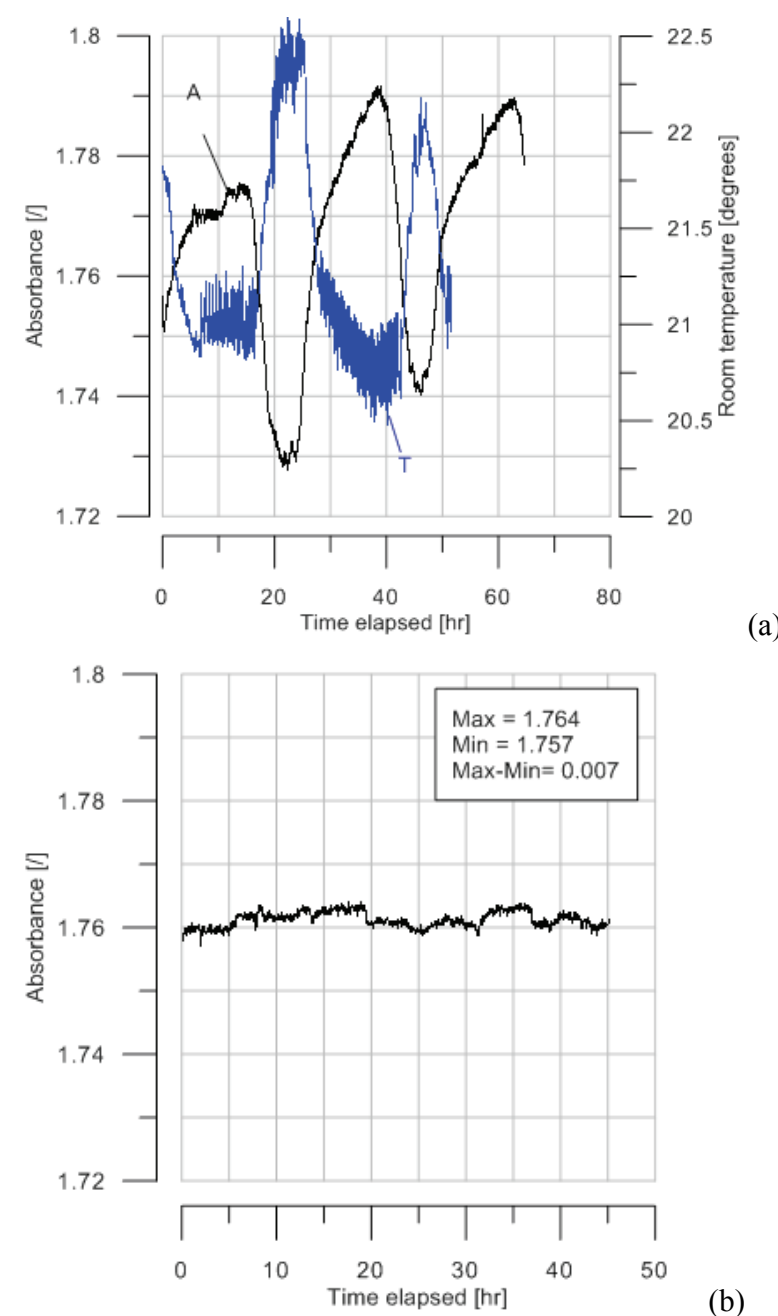

(a)

Figure 3. Results of the drift experiment: evolution of room temperature and absorbance with time with the laser and ITC controller outside (a) the temperature controlled environment. (b): evolution of absorbance with all equipment placed inside the temperature controlled environment.

Estimating the error made on the actual PEG concentration requires an assessment of the goodness of fit of the calibration curve, i.e. the best fit of the calibration data. The data showed in Figure 2 can be fitted with the following equation:

$$
C=-0.726 A+1.33
$$


where $A$ is the absorbance of the cuvettes + PEG solution, and $C$ is the PEG concentration. Such a fit returns a $\mathrm{R}^{2}$ of 0.988 and a residual mean square of $2.98 \times 10^{-4}$.

The standard error being the square root of the residual mean square, it yields an absolute error of $\pm 0.017 \mathrm{gr}$ PEG/gr water. At the highest concentration of PEG (0.9 gr PEG/ gr water, see the Brix Index - concentration data by the authors in Figure 4), this represents a relative error of $\pm 2 \%$.

In comparison, the E Line 90 hand held refractometer by Bellingham + Stanley Ltd has a resolution of 0.2 unit of Brix Index (absolute error of \pm 0.1 unit of Brix Index) but the absolute error associated with the goodness of fit of the "Concentration-Brix Index" data is about $0.11 \mathrm{gr}$ $\mathrm{PEG} / \mathrm{gr}$ Water (from a residual mean square of 0.0117 , Figure 4). At the highest concentration of PEG (0.9 gr $\mathrm{PEG} / \mathrm{gr}$ water), this represents a relative error of $\pm 12 \%$.

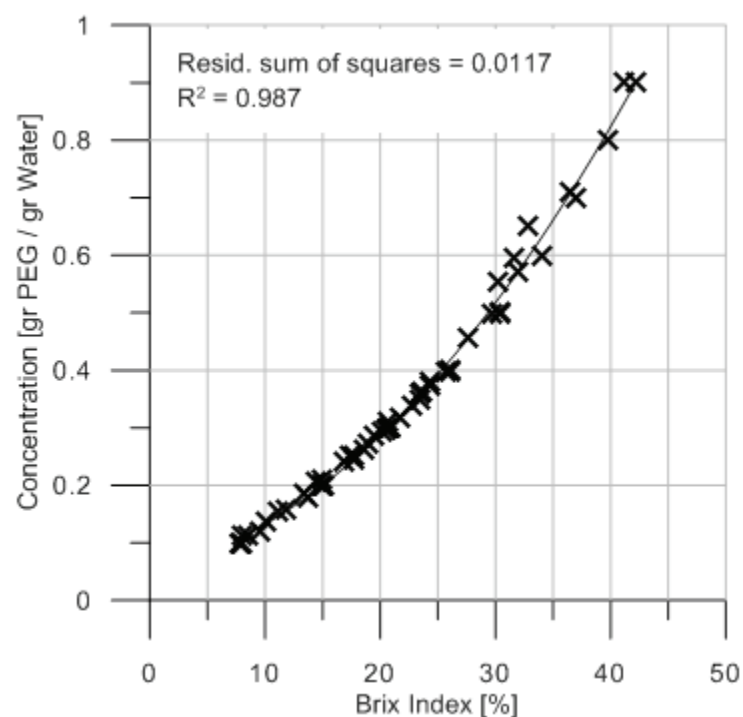

Figure 4. Experimental correlation between concentration of PEG solution and Brix Index measured with a hand held refractometer.

The standard error, as estimated from the residual mean square is an average value and does not necessarily reflect the actual accuracy of measurement, especially at the low end of concentrations.

In order to better assess accuracy at low concentrations, a detection test was performed, where a small amount of water was added to a PEG solution in order to reduce its concentration from 0.0562 to $0.0558 \mathrm{gr} P E G / \mathrm{gr}$ water. This is a difference of concentration of $0.0004 \mathrm{gr}$ PEG/gr water. The absorbance changed from 1.759 to 1.803 , which represents in increase of 0.044 , well beyond the equivalent resolution of the sensor of 0.0057 (see section 4.2). This result suggests that the change in concentration was detected by the sensor.

In conclusion, this new sensor provides an indirect measurement of PEG concentration via absorbance measurement with a much better accuracy than hand held refractometer.

\subsection{Test series \#3}

The drift experiment (Figure 3) demonstrated that temperature fluctuations do influence the absorbance measurement of a given PEG solution. Hence, additional tests were conducted at about 23, 26 and 29 degrees to assess this effect. Effectively, the results confirm the temperature sensitivity. Figure 5 shows that the absorbance progressively increases when the temperature rises. The magnitude of the increase is in the order to $8 \%$ for 9 degrees of heating (from 20 to 29 degrees). Such variations are consistent with data that can be found in the literature [6].

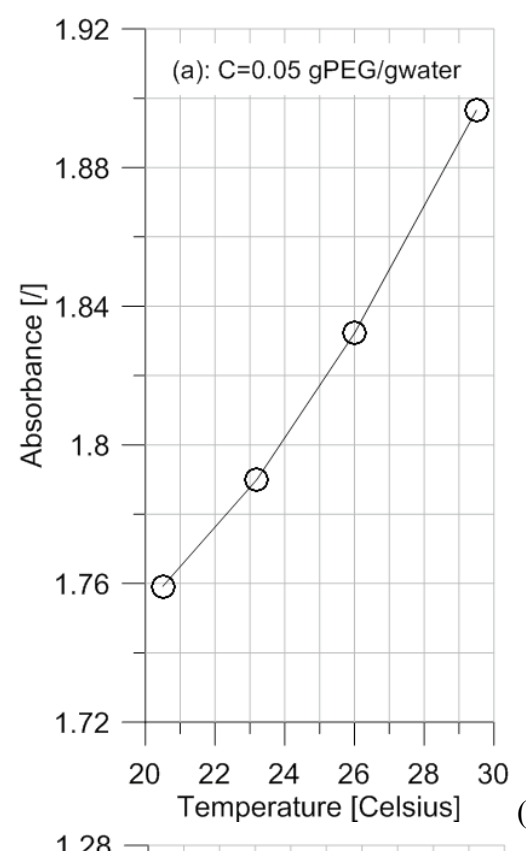

(a)

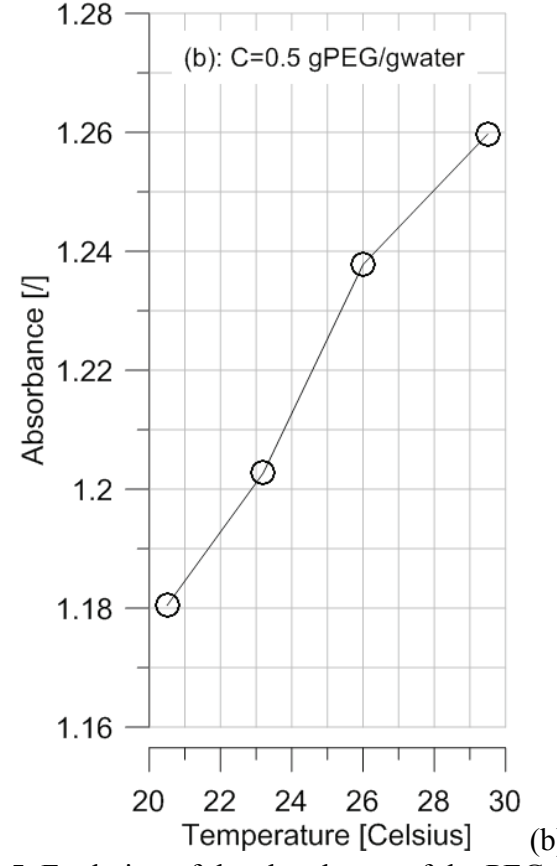

Figure 5. Evolution of the absorbance of the PEG solution with temperature. (a): $\mathrm{C}=0.05 \mathrm{~g} \mathrm{PEG} / \mathrm{g}$ water, (b): $\mathrm{C}=0.5 \mathrm{~g}$ PEG/g water. 


\subsection{Test series \#4}

Soil specimens often have chemical species dissolved in their pore water. The semi permeable membranes typically used for the osmotic method do not typically prevent these chemical species (cations and anions) from diffusing into the PEG solution because their molecular weight cut off is too high. Consequently, it is possible to find some cations and anions in the PEG solution post testing [5]. Test series \#4 was conducted to assess whether these cations and anions could interfere with the measurement of PEG concentration with the new sensor. The absorbance measurements made in seawater are plotted in Figure 6 together with the line representing the best fit of the data showed in Figure 2. Note that the concentration values were corrected to be expressed in $g$ $\mathrm{PEG} / \mathrm{g}$ of water. With the seawater composition given in Table 2, $0.5 \mathrm{~g} \mathrm{PEG} / \mathrm{g}$ seawater is equivalent to $0.518 \mathrm{~g}$ $\mathrm{PEG} / \mathrm{g}$ water. Figure 6 shows that the presence of cations and anions (at a relatively high concentration compared to typical pore water) does not affect the measurement of PEG concentration in a significant manner. The data points do not fall exactly on the line but this is attributed to natural scattering as visible in Figure 2.

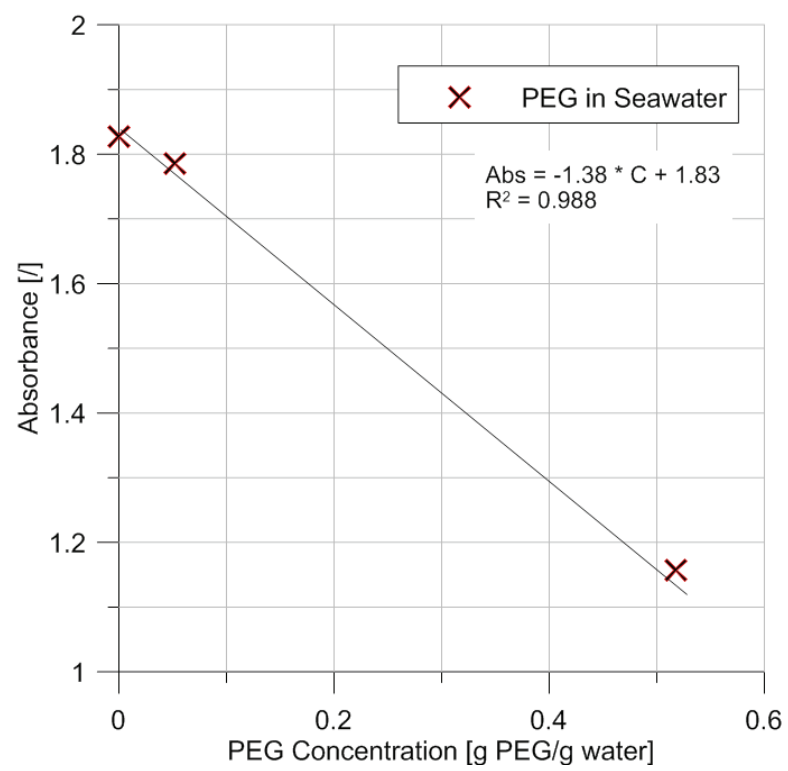

Figure 6. results of absorbance for PEG20k dissolved in seawater. The line represents the best fit of all data presented in Figure 1. Equation of the line is: $\mathrm{Abs}=-1.38 \mathrm{C}+1.83$

\section{Conclusions}

This paper presents the validation and characteristics of a new sensor for the indirect measurement of PEG concentration. The sensor uses a laser, the intensity of which is absorbed by the water in the PEG solution. The input and output voltages are used to compute the absorbance, a quantity shown to be strongly correlated to the PEG concentration. The equivalent resolution of the new sensor is \pm 0.0057 while the error on the estimation of concentration is about $\pm 2 \%$ at the high end of PEG concentration. A temperature dependence was observed and quantified. Finally, the tests conducted suggest that the chemical species that could diffuse from the soil to the PEG solution are unlikely to interfere in a significant manner with the measurement of PEG concentration.

\section{References}

1. G. Kassiff and A. Ben Shalom Géotechnique, 21, 245-255 (1971)

2. P. Delage, M. Howat and Y.J. Cui. Eng. Geology, 50 (1-2) : 31-48 (1998).

3. R. Monroy, A. Ridley, K. Dineen, L. Zdravkovic (2007). ASTM Geotech. Test. J. 30, 1-7.

4. J. Lagerwerff, G. Ogata and H. Eagle, Science (80). 133, 1486-1487 (1961).

5. M. Tadza PhD Cardiff University (2011).

6. J.R. Collins. Phys Rev. 26, 771-779 (1925) 